\title{
Conceptual Metaphor and Dual Coding on Abstract Concepts
}

\author{
Xudong $\mathrm{Ma}^{1, *}$, Beilin $\mathrm{Bao}^{2}$, and Xinrui $\mathrm{Li}^{3}$ \\ ${ }^{1}$ School of Art and Science, Rutgers University-New Brunswick, New Brunswick, NJ 08901, US, 1054618445@qq.com \\ ${ }^{2}$ Yangzhou High School of Jiangsu Province, Yangzhou, Jiangsu 225009, China, bbl12345bbl@ 163.com \\ ${ }^{3}$ Woming High School, Kunming, Yunnan 065100, China, 798206176@qq.com
}

ABSTRACT

As a currently dominant theory that explains how human beings process concepts and ideas mentally, the Embodied Cognition theory has met difficulty in explaining how abstract concepts are embodied. In summary, unlike concrete concepts, it is seemingly hard to relate abstract concepts with perceptuo-motor simulations. A series of theories were proposed in recent decades aiming at solving this difficulty, and the Conceptual Metaphor Theory is a majorly influential one of them. This theory supposes that human beings construct multiple kinds of metaphors to relate abstract concepts with perceptuo-motor simulations. Later, however, it is indicated that the Conceptual Metaphor Theory also has difficulties in explaining the embodiment of abstract concepts. This paper does a more specific introduction to the Conceptual Metaphor Theory and what difficulty it meets in explaining the embodiment of abstract concepts. Then, another majorly influential theory, the Dual-Coding Theory, and how it proposes an alternative solution to the problem posed by abstract concepts is reviewed. Finally, this paper makes a proposal as an attempt to conclude an essential prediction that needs to be tested to verify the effectiveness of the Dual-Coding Theory in explaining the embodiment of abstract concepts.

Keywords: Embodied Cognition, abstract concept, Conceptual Metaphor Theory, Dual-Coding Theory.

\section{INTRODUCTION}

For decades, the debate on how the human brain works to process meanings and concepts has never been ceased. In the middle of the stage, there stands the Embodied Cognition theory (EC), on which a dominant amount of argument is focused. This theory was greatly promoted by important evidence found to implicate the activation of perception, action, and emotion systems in conceptual tasks [1] and thus led to the following enormous development of the so-called "embodied" view of concepts. In summary, the EC states that affective and sensorimotor systems in the human brain play a central role in cognitive tasks that process concepts and meanings. This description suggests the operation of modality-specific simulations, in which concepts and meanings are instantiated through reenactments.

In fact, such a procedure of processing conceptual tasks has been proved by a series of experiments and observations. Gonzalez and his fellow colleagues [2] conducted an experiment to let participants read multiple groups of words in different types silently and meanwhile scan their brain activities through an fMRI machine to see how their brains react to the readings. In all word groups, olfactory words, such as CINNAMON, COFFEE, LEMON, etc, and neutral words, such as PIANO, COAT, NEEDLE, etc, are mixed up evenly. What's Surprising, the result reveals that typical olfactory brain areas are activated when the participants are merely reading olfactory words but not smelling anything. This study demonstrates the operation of sensorimotor systems during conceptual tasks. Further studies have also found evidence for other kinds of embodiment. For example, there is evidence that people mentally represent the shapes of objects while comprehending the meaning-related words [3]; also, people are found to be oriented object words to the objects' typical locations, for example, top of the body while reading the word, HEAD [4]. Overall, as more and more evidence of embodiment has been observed, the EC was placed in a more and more dominant position to explain the human cognitive mechanism of conceptual processing.

However, challenges posed for the EC soon arise. Although there is an enormous amount of evidence that concepts are embodied in the human mind, these pieces 
of evidences merely demonstrate how concrete concepts are embodied. There is another type of concept that these pieces of evidence did not prove to be embodied, they are abstract concepts. The division between concrete concepts and abstract concepts is crucial. Roughly speaking, concrete concepts are physical and normally related to or interpreted by approachable things in the physical world. For example, words like THROW, THICK, LEG are concrete. Reversely, abstract concepts are not physical, but rather ideological. They normally can not be related to or interpreted by approachable things in the physical world. For example, words like LIBERTY, SOCIETY, LOVE are abstract. It is easy to see how concrete concepts can be embodied, since there is a explicit connection between these concepts and human physical activities that relate to perception and action. But it is very difficult to see how abstract concepts can be embodied, as these concepts are non-physical and can hardly be related to human perception and action. This difficulty remains until people found that metaphors might be used to explain the embodiment of abstract concepts. The conceptual metaphor theory (CMT) suggests that people use different kinds of metaphors to interpret the abstractness of abstract concepts in physical ways [5]. For example, people always relate the abstract concept of GOOD to the physical position of TOP by saying things like: "Jon was ranked the top in his class for the final exam!" The CMT indicates that people use metaphor to talk when they need to express abstract things, and this poses the possibility that people might also think metaphorically and thus get those abstract concepts embodied.

This possibility soon raised a big trend of researches. Some evidence is found that people really think metaphorically. Lawrence E. Williams and John A. Bargh [6] found that people's concept of interpersonal warmth can be promoted by merely experiencing physical warmth. Such evidence all strengthened people's belief that abstract concepts are embodied through conceptual metaphors. However, afterward, Daniel Casasanto and Tom Gijssels [7] reviewed different pieces of evidence and pointed out that though there is evidence that people think metaphorically, it does not entail that these conceptual metaphors of abstract concepts are embodied. There should not be drawn an equivalence between thinking metaphorically and abstract concepts being embodied. Even though people might really think metaphorically, it is still possible that these metaphors are be embodied, and fMRI scanning has to be conducted to see if the metaphors are really embodied and activate related perceptual and sensorimotor brain systems. In fact, there has been found that conceptual metaphors of abstract concepts are not embodied. Quadflieg and his colleague [8] conducted an experiment to ask participants to distinguish "high" V.S. "low" stimuli of four kinds while they underwent an fMRI scanning. The result revealed that when participants meet "high" and "low" words of the abstract type, such as words with positive valence like LAUGHTER and words with negative valence like DANGER, their corresponding perceptual and sensorimotor areas are not activated as observed by the fMRI machine.

Thus, a new difficulty is now posed upon the feasibility of using the CMT to explain how abstract concepts can be embodied. The CMT might just fail to work for explaining the embodiment of abstract concepts. Thus, in this paper, other kinds of theories proposed and studied by different groups of researchers that aim to solve the disembodiment problem of abstract concepts will be reviewed. One of these theories, that is, the embodied Dual Coding Theory (DCT), will be mainly focused on. This paper will describe what the theory suggests and different branch versions of it. Then, an evaluation will be done on an experiment that has been considered as evidence for the embodied Dual Coding Theory. Finally, a proposal on how the embodied dual-code theory can be further tested will be proposed.

\section{THE EMBODIED DUAL CODING THEORY}

As severe challenges have been posed against the feasibility for using the CMT to explain solve the disembodiment problem of abstract concepts, an alternative theory has started to earn increasing attention, that is, the Dual Coding Theory (DCT) [9]. This theory explains the concreteness effect with the higher imageability of concrete concepts comparing to abstract concepts. According to DCT, all concepts are represented through the verbal system, but only concrete concepts have a direct connection with images. For instance, the abstract concept of DANGER evokes images only through the mediation of concrete concepts such as KNIFE.

Along with the ongoing studies, the original version of DCT inspired researchers to posit further possibilities that concepts can be dual-coded. These possibilities then evolve to further different branch versions of the DCT, which put more focuses on addressing the disembodiment problem. Two of the branch versions of DCT are the language-and-situated-simulation (LASS) theory [10] and the word-as-tool (WAT) theory [11]. According to the LASS theory, language comprehension involves the continuous interaction between the processing of linguistic forms and situated simulations. On the other hand, the WAT theory suggests that linguistic forms are not merely a means of engaging simulations but a means of leveraging people's social experience. This theory further suggests that abstract concepts require longer-lasting social interaction than concrete concepts to be comprehended, and this leads to their heavier reliance on linguistic simulation. In 
summary, both of these two branch versions suggest that the internalized language in the human mind serves as an additional conceptual format. The internalized language that talks in the inner mind activates either a situated simulation or our preceding social experience related to the encountered abstract concept.

As discussions on the DCT are ongoing, further evidence that prove the feasibility for DCT to addressing the disembodied problem of abstract concepts are found. One of them that will be evaluated in this paper focuses on observing the activation of language systems in the human brain that helps people comprehend abstract concepts. This experiment is conducted by Katrin Sakreida and her colleagues [12]. In the experiment, they ask participants to read 30 sentences that are visually presented silently. In the meantime, participants are required to press a button as fast as possible if the sentence they read refers to an action that is typically performed with the foot and/or leg, for example, when they see the word, KICK. During the task, an fMRI scanning is processed. Also, importantly, in those 30 sentences, four types of noun-verb combinations are included. They are 1 . noun referring to a graspable object/motor verb, 2. noun referring to a graspable object/non-motor verb, 3. noun referring to a non-graspable entity/motor verb, and 4 . noun referring to a non-graspable entity/non-motor verb. These four types of noun-verb combination are constructed according to the concrete-abstract property of the word, and so the pairings are actually divided as 1. concrete noun/concrete verb, 2. concrete noun/abstract verb, 3. abstract noun/ concrete verb, and 4. abstract noun/abstract verb. Finally, the fMRI scanning result of this experiment shows that concrete stimuli revealed activation to the sensorimotor system whereas abstract stimuli elicited pronounced activation of areas that are known to underlie lexical and phonological processing.

This experiment demonstrates that while encountering concrete and abstract words, people activate different types of combinations of brain systems and neural functions respectively to code the concrete concepts and abstract concepts in different ways. As abstract concepts elicit activation of language systems but not sensorimotor systems, the possibility that language has a symbolic property that serves as a conceptual format and helps people activate situated simulation or preceding experience is suggested. Thus, the result of Katrin Sakreida's study is considered to be strong evidence that supports both the LASS theory and the WAT theory.

\section{DISCUSSION: A PROPOSAL TO THE DCT}

Along with more and more supports from various evidence, the DCT earned stronger credits from a number of researchers. The possibility to use the DCT to address the disembodiment problem posed by abstract concepts is enlarged. Thus, follow-up examinations and suggestions on how the DCT can be adopted and updated are urged to be taken. In this section, this paper aims to indicate the central model described by the DCT and how it can be further verified. In detail, this paper will propose a specific way of designing an experiment to suggest how one of the branch versions of the DCT, the word-as-tool theory, can be examined and demonstrate how experiments that aim to seek support for the DCT should be designed.

First of all, remember that all branch versions of the embodied DCT share the idea that internalized language has a symbolic property that serves as an additional conceptual format. For example, the word-as-tool theory states that abstract concepts require longer-lasting social interaction than concrete concepts to be acquired, and this leads to their greater reliance on linguistic simulations. In this way, the DCT proposes a negative relationship between abstractness and imageability: the more abstract a concept is, the less imageable it is, and so, in the view of WAT theory, the greater reliance on linguistic simulations it has. Such a description of the DCT portrays a proportional relationship that underlies the theory itself. In other words, in central task to verify the feasibility of the DCT is to verify if such a proportional relation does exist. If evidence that suggests that abstract concepts activate of language systems but not sensorimotor systems, but meanwhile it fails to demonstrate such a proportional relation, then this evidence does not essentially prove the feasibility of the DCT. Moreover, the verification on different subjects in the proportional relation directly enables researchers to examine which branch version of the DCT is a closer one to the truth. For example, the proportional relation that the WAT theory posits is between the amount of social interaction time for an abstract concept to be acquired and its reliance on linguistic simulation. Thus, based on different kinds of branch version of the DCT, the experimental design should be different and the comparison between this one and others should be highlighted.

Then, this paper focuses on suggesting one experimental design that specifically aims to examine the proportional relation posited by the WAT theory. As mentioned above, the WAT theory posits a positive proportional relation between the amount of social interaction time for an abstract concept to be acquired and its reliance on linguistic simulation. Such a relation presupposes that there is a significant difference between abstract concepts, that is, different abstract concepts, or even concrete concepts, might need a different amount of social interaction time to be acquired. Such a presupposition provides a great intuition for researchers to categorize abstract concepts and concrete concepts to test if people have different 
brain reactions to them. This work of categorization, seemingly, requires a great number of observations to support the result. Thus, in this paper, only an intuitive suggestion will be provided.

The level of the amount of social interaction time required to learn an abstract concept may relate to one factor, that is, roughly speaking, the semantic complexity of the concept. It is normal to see that one word can be underlaid or explained by a group of other words. For example, MILK can be explained as WHITE FLAVORFUL LIQUID. Such a property that a word can be subdivided into multiple words might reveal the level of complexity of that word. Nonetheless, such a subdivision is not endless, for example, intuitively, LIQUID is much more difficult to be subdivided into a group of other words to be explained comparing to MILK. In this sense, it can be concluded that the word MILK is more complex than LIQUID. Then, such a categorization of semantic complexity, if further experiments and observations are taken properly, might be one of the factors that underlie the amount of social interaction time required for a concept to be acquired. Hence, then, further categorization of abstract concepts can be done. For example, in the method that is demonstrated above, it can be inferred that the abstract concept, GOOD, is less complex than the abstract concept, LIBERTY, since LIBERTY can be explained as a good property that underlies human rights and it is intuitively more difficult to do such a subdivision on GOOD. Again, with a presumably approachable amount of experiments and tests, such kinds of categorization between GOOD and LIBERTY can be established, and so one subject of the proportional relation proposed by the WAT theory is founded for further experiments.

Afterward, the only thing left is simply to examine if the proportional relation that abstract concepts with different levels of semantic complexity have different levels of reliance on linguistic simulation. Such a relation can be examined by conducting fMRI scanning. For example, researchers can first categorize a group of words into different levels of semantic complexity according to the method mentioned above. One group is made up of words like LIBERTY, DEMOCRACY, FREEDOM that is semantically more complex, another group with words like GOOD, KINDNESS, EVIL that are semantically less complex. Then, let participants read these words respectively and meanwhile take an fMRI scanning to see how language systems of their brain react. If the group of words that are semantically more complex raises stronger activation of the language system in the participant's brain than the group of words that are semantically less complex, then it will be proved that the more amount of social interaction time required for the concept to be acquired, the stronger the reliance this concept has on linguistic simulation. And so the WAT theory will be examined and verified.

\section{CONCLUSION}

In sum, as the feasibility for the Conceptual Metaphor Theory to explain how abstract concepts can be embodied is severely challenged, the Dual Coding Theory might be a good alternative theory for people to test the feasibility for it to address the disembodiment problem. Indeed, more and more aspects of the DCT are examined, and more and more researchers are await to conduct further researches for such feasibility. Now, undoubtedly, cognitive science is approaching closer and closer to the day it unmasks the secret of how meanings and concepts are processed in the human mind. As this paper proposes to do experiments and categorizations of the semantic complexity of both abstract and concrete concepts, this proposal opens for further development and discussion.

\section{REFERENCES}

[1]. Barsalou, L. W. (1999). Perceptual symbol systems. Behavioral and Brain Sciences, 22, 577609. doi:10.1017/S0140525X99002149. disc. 609660.

[2]. González, J., Barros-Loscertales, A., Pulvermüller, F., Meseguer, V., Sanjuán, A., Belloch, V., \& Ávila, C. (2006). Reading cinnamon activates olfactory brain regions. Neuroimage, 32(2), 906-912.

[3]. Zwaan, R.A., Stanfield, R.A. \& Yaxley, R.H. (2002). Language comprehenders mentally represent the shapes of objects. Psychological Science, 13, 168-171.

[4]. Estes, Z., Verges, M., \& Barsalou, L. W. (2008). Head up, foot down: Object words orient attention to the objects' typical location. Psychological Science, 19(2), 93-97.

[5]. Lakoff, G., \& Johnson, M. (1980). Metaphors we live by. Chicago, IL: University of Chicago Press.

[6]. Williams, L. E., and Bargh, J. A. (2008). Experiencing physical warmth promotes interpersonal warmth. Science, 322, 606-607.

[7]. Casasanto, D. \& Gijssels, T. (2015). What makes a metaphor an embodied metaphor? Linguistics Vanguard. DOI: 10.1515/lingvan-2014-1015

[8]. Quadflieg, Susanne, Joset A. Etzel, Valeria Gazzola, Christian Keysers, Thomas W. Schubert, Gordon D. Waiter \& C. Neil Macrae. 2011. Puddles, parties, and professors: Linking word categorization to neural patterns of visuospatial coding. Journal of Cognitive Neuroscience 23(10). 2636-2649.

[9]. Paivio, A. (1986). Mental representations: A dual 
coding approach. New York, NY: Oxford University Press.

[10].Barsalou, L. W., Santos, A., Simmons, W. K., \& Wilson, C. D. (2008). Language and simulation in conceptual processing. In M. De Vega, A. M. Glenberg, \& A. C. Graesser (Eds.), Symbols and embodiment: Debates on meaning and cognition (pp. 245-284). New York, NY: Oxford University Press.

[11].Borghi, A. M., \& Cimatti, F. (2009). Words as tools and the problem of abstract words meanings. In N. Taatgen \& H. van Rijn (Eds.), Proceedings of the $31^{\text {st }}$ Annual Conference of the Cognitive Science Society (pp. 2304-2309). Austin, TX: Cognitive Science Society.

[12].Sakreida, K., Scorolli, C., Menz, M. M., Heim, S., Borghi, A. M., \& Binkofski, F. (2013). Are abstract action words embodied? An fMRI investigation at the interface between language and motor cognition. Frontiers in Human Neuroscience, 7(125), 1-13. doi: 10.3389/fnhum.2013.00125 